Original Research Article

\title{
Application of Photoelectric Detection Technology in Nondestructive Testing
}

\author{
Xiumin Jiang,Xianhua Tang, Yanlin Peng \\ Department of Imaging, Affiliated Hospital of Jilin Medical University, Jilin, China
}

\begin{abstract}
Non-destructive testing, is the use of sound, light, magnetic and electrical characteristics, without prejudice to or does not affect the performance of the object being tested under the premise of the detection of the existence of defects or non-uniformity, and gives the general term for all technical means of the size, location, nature and quantity of the defect. As does not affect the performance of the object being tested, non-destructive testing technology in these years has been rapid development. Photoelectric detection technology is one of the main technologies of photoelectric information technology. It is based on modern optoelectronic devices such as laser infrared fiber. The optical radiation of the detected object is received by the photoelectric detector and converted into electrical signal, and then processed by the following, access to useful information technology. The combination of photoelectric detection technology and nondestructive testing technology can take the advantages of both, and it will be widely used. In this paper, the commonly used non-destructive testing technology based on photoelectric technology is summarized. Mainly discusses infrared detection technology, machine vision detection technology, x-ray detection technology and other non-destructive testing technology. Their principles and scope of application are discussed in detail, and examples of the application of each technology in real life.
\end{abstract}

KEYWORDS: photoelectric detection, nondestructive testing, infrared imaging, machine vision, $\mathrm{X}$ - ray inspection

\section{Photoelectric detection technology and non-destructive testing}

\subsection{Photoelectric detection technology principle}

Optoelectronic detection technology is one of the main technologies of photoelectric information technology. It is based on modern optoelectronic devices such as laser, infrared and optical fiber. By means of optical radiation of the object to be detected, optical radiation is received by photoelectric detector and converted into electrical signal, by the input circuit, amplification filter detection circuit to extract useful information, and then by the analog/ digital conversion interface input computer computing processing, and finally display the output required to detect the physical quantity and other parameters.

Photoelectric detection technology mainly includes photoelectric conversion technology, optical information acquisition and light measurement technology and measurement information of photoelectric processing technology. Mainly has the following characteristics:

1. High precision laser interferometry to measure the length of the accuracy of up to $0.05 \mathrm{um} / \mathrm{m}$; with laser range measurement method to determine the distance between the earth and the moon can reach $1 \mathrm{~m}$.

2. High speed photoelectric detection technology to light as the medium, and the light propagation speed is very fast, no doubt use optical methods to obtain and transmit information is the fastest.

3. Distance from a large range. Light is the most convenient long-distance transmission of the medium, especially for remote control and telemetry, such as photoelectric tracking.

4. Non-contact measurement. Light to the measured object can be considered no measurement of force, and therefore no friction, can achieve dynamic measurement, is the most efficient measurement methods.

Copyright (C) 2017 -. This is an Open Access article distributed under the terms of the Creative Commons Attribution-NonCommercial 4.0 International License (http://creativecommons.org/licenses/by-nc/4.0/), permitting all non-commercial use, distribution, and reproduction in any medium, provided the original work is properly cited. 


\subsection{The status of photoelectric detection technology}

With the rapid development of science and technology, photoelectric detection technology has developed nanometer, sub-nano high-precision optical measurement of new technologies; small, fast micro-light, machine, electrical detection system in various fields more and more widely. Non-contact, fast on-line measurement has replaced the original contact, slower detection technology, and to the micro-space three-dimensional measurement technology and large space threedimensional measurement technology direction; closed-loop control of the photoelectric detection system to achieve photoelectric measurement and photoelectric control integration to the areas that people cannot reach. Photoelectric tracking and photoelectric scanning measurement technology and other advanced optoelectronic detection technology advances and extensive application of people's lives, industrial production and even national defense science and technology have a huge impact and reform. With the rapid development of optical fiber sensing technology, optical fiber gas sensor has also been widely studied and applied. It has the advantages of high sensitivity, fast response, explosionproof and explosion-proof, free from electromagnetic interference, long-distance transmission of optical signal and realtime telemetry and so on. Therefore, the research on photoelectric detection method has been increasing.

With the wide application of fiber grating sensors, fiber grating sensor signal detection system has also been a great development. Compared with the traditional monochromator, spectrometer scanning and other ways to detect the fiber grating sensor signal way, the use of photoelectric conversion method, that is, the measurement of light intensity signal for the voltage signal measurement has its advantages, It has the advantages of low cost of manufacturing equipment, easy to carry, can achieve real-time changes in signal high-speed detection and have a wider range of engineering applications and so on.

Photoelectric detection technology in human genetic engineering also has a wide range of development, such as the use of visible light signal changes in the detection of DNA hybridization signal. This new DNA photodetection system is also composed of a self-assembled silicon integrated circuit with a compatible probe DNA. In the system, the target DNA sequence is connected with the magnetic bead, and when the target DNA sequence hybridizes with the probe DNA molecule, the magnetic bead will cover the dark surface of the carrier surface. And the photodiode array beneath the carrier can detect the optical signal changes before and after the DNA hybridization reaction and output the DNA hybridization signal.

The new anti-jamming type photoelectric detection head can effectively reduce the electrical interference noise and improve the anti-jamming performance and working reliability of the detection head by optimizing the working parameters of the infrared emission tube and using the pulse gating gated detection circuit. The experimental data show its effectiveness. At the same time, the kind of photoelectric detection head has been in a number of large-scale mechanical and electrical equipment manufacturers' application, the factory actual application results show that: the new type of photoelectric detection head error rate of less than one thousandth of old products, anti-electrical interference significantly improved, with the industrial high reliability.

\subsection{Overview of Non-destructive Testing Technology}

NDT (Non-destructive testing), is the use of sound, light, magnetic and electrical characteristics, without prejudice to or without affecting the performance of the object under the premise of the test object is detected in the defect or nonuniformity, The general term for all technical means of giving the size, location, nature and quantity of the defect. NDT refers to the material or the work-piece to carry out a non-damage information, and then determine the subject of the technical status of the object, such as qualified or not, the remaining life or not affect its future performance or use of detection means.

Through the use of NDT, can be found in the material or the work-piece inside the surface and the existence of defects, can measure the geometric characteristics of the work-piece and size, and can measure the material or workpiece internal composition, structure, physical properties and status. Non-destructive testing methods can be divided into conventional non-destructive testing methods and unconventional non-destructive testing methods. Conventional non-destructive testing methods are ultrasonic testing, radiographic testing, magnetic particle testing, penetrate testing, Eddy current testing, Unconventional non-destructive testing acoustic emission, leak testing, photogrammetry optical holography, infrared thermal imaging, microwave testing.

With the rapid development of these years, non-destructive testing technology gradually developed from the qualitative detection to the quantitative detection direction. In the detection process, not only the presence or absence of the defect is detected, but also the type, size, shape and orientation of the defect.

Through the above analysis, we can find that the essence of photoelectric detection technology and non-destructive testing technology is to obtain the state information of the object through the sensor. Through the sensor to detect the electrical signal, after the subsequent processing, resulting in the state of the object information to determine the operation of the object state. From this level to judge, the two should have a great intersection, the fact is the case, 
photoelectric detection technology in the field of non-destructive testing has been widely used, including infrared imaging technology, machine vision technology and x-ray technology.

\subsection{The development trend of photoelectric detection technology}

With the fierce competition in the world is the rapid pace of rapid progress and scientific research and technology to improve the detection technology in all sectors of the national economy plays a decisive role, regardless of scientific research, product quality and automatic control need to detect the use of modern optoelectronic technology as a means of detection, with no contact, lossless, long distance, anti-interference ability, by the environmental impact of small, fast detection, high precision measurement accuracy is the main direction of today's detection technology development. Photoelectric detection technology will be toward high precision, intelligent, digital, diversified, miniaturization, automation direction. The high-precision means that the detection accuracy to the direction of high-precision development, Nano high precision optical measurement of new technology is the future development of hot; intelligent refers to the detection system to the intelligent direction, such as photoelectric tracking and photoelectric scanning measurement technology digitalization refers to the detection results to the digital, to achieve the development of optoelectronic measurement and photoelectric control integration; diversification refers to the detection of photoelectric detection equipment to comprehensive, multi-parameter, multi-dimensional measurement and other diversified direction, and to people cannot touch the development of micro-technology, such as micro-space three-dimensional measurement technology and large-space three-dimensional measurement technology; miniaturization refers to the electronic components used in photoelectric detection equipment and circuit to the integrated direction of development; miniaturization refers to the photoelectric detection system toward small, automation, refers to the detection technology to the development of automation, non-contact, rapid online measurement, detection of the state to the dynamic measurement of the direction of development.

Laser-based optoelectronic detection system has become the most important direction of development. With the increase in precision and functional characteristics, it will continue to replace those expensive and complex detection program. As the laser technology continues to improve and the stability of the cost of decline, it is certain that laser optoelectronic detection technology will increasingly become the preferred optoelectronic users program. Laser ultrasonic detection technology is another important development direction of photoelectric detection technology. This technique utilizes the high-energy laser pulse and the instantaneous thermal action of the surface of the material to produce a thermal characteristic region on the solid surface, and then uses this small thermal layer to generate thermal stresses around the thermal expansion and diffusion around the material, resulting in this thermal stress ultrasonic. Compared with the conventional ultrasonic detection method, laser ultrasonic technology has the following advantages: laser ultrasound does not require coupling agent, to avoid the coupling agent on the measurement range and accuracy of the impact; laser ultrasound can achieve long-distance operation can be used for high temperature environment and corrosive, Radioactive and other harsh conditions, and can achieve rapid scanning, the rapid production of the scene of the work-piece on-line detection; laser ultrasound blind area is less than 100 microns, can be used to measure thin parts. The frequency of laser ultrasound is wider than that of conventional transducers and has the ability to measure tiny crack. Laser ultrasound can be used for complex and restricted geometrical shapes, such as small diameter pipes and so on. The spatial resolution is high. It facilitates the precise positioning and dimensional measurement of defects and can be used as a sound source in theoretical research.

Intelligent photoelectric detection system has also become another very important direction of development. Intelligent photoelectric detection system consists of intelligent signal processing system, photoelectric sensor system, measurement and control system, out-put system and interface unit and so on. It integrates optical acquisition, optical transformation, photoelectric conversion, circuit conditioning, peripheral interface and information output. It can realize optical information acquisition, photoelectric signal conversion, signal detection, logic operation and reasoning, memory storage and information transmission and other functions, and automatically complete self-test and self-diagnosis and adjustment and other functions. Intelligent photoelectric detection system due to the ability to adapt to the environment, a wide range of measurement, measurement accuracy, especially to strengthen the artificial intelligence system, you can automatically on the noise, temperature, voltage fluctuations and changes in light source correction, coupled with good human-computer interaction Interface, greatly simplifies the operating procedures, improve the efficiency of numerical processing and analysis.

\section{Non-destructive testing technology for infrared imaging}

\subsection{Infrared imaging principle}

Infrared is between the visible and microwave electromagnetic waves, its wavelength range of $0.77-1000 \mathrm{um}$, the frequency. In nature, any object above absolute temperature is an infrared radiation source with a radiation phenomenon. Stephen-Boltzmann's Law tells us that the intensity of the emitted infrared rays is: 
Which is the gray body emission coefficient, for the Stephen - Boltzmann constant, $\mathrm{T}$ is the absolute temperature of the object.

Infrared non-destructive testing is to measure the heat and heat flow through the object, when there are cracks or other defects within the object, it will change the heat conduction of objects, so that the surface temperature distribution of objects appear uneven or uneven changes, the use of these differences or uneven change the image, can be intuitively detect the defective position of the object. Of course, there will be a variety of specific heat input.

It can be seen from Figure 4.2 that when there is a defect in the object, the amount of heat passing through the object and the amount of reflection by the object are no longer evenly distributed, and the infrared radiation from the point of the measured object is perpendicular and horizontal Scanning the mirror, through the vertical and horizontal scanning mirror gathered to the infrared detector, the infrared energy signal into electrical signals, amplified by the amplifier and signal processor processing. The processed signal reflects the infrared image signal of the surface temperature field of the measured object. This infrared image can directly reflect the existence of quality defects such as the measured object.

Infrared imaging technology is usually used to detect the quality of metal or non-metallic materials, detection of internal defects, especially for the detection of welding defects, there are other detection methods cannot match the advantages.

\subsection{Detection of welding defects}

Machinery and equipment, some metal structures due to welding and other reasons failed quality, thus affecting the mechanical strength and service life, and even lead to accidents. The detection of welding defects is the key to ensure the quality of welding, but the welding surface is uneven, using radiation, ultrasonic, eddy current and other methods are difficult to find internal defects; and infrared thermal imaging technology is not subject to the surface shape of the object, quickly and accurately detect the various defects in the welding area. The specific principles have been discussed in detail in front, but here the heat source uses the heat to heat. The actual section of the weld zone is shown in Figure 4.3, (a) the weld zone is defect free, and (b) the weld zone is defective. If an AC voltage is applied to both ends of the weld zone, there will be alternating current through the weld, due to the current skin effect, near the surface of the current than the lower. Due to the role of current, the weld will produce a certain amount of heat, the size of the heat is proportional to the material resistivity and current density of the square. In the absence of defects in the welding area, the current distribution is uniform, the heat generated everywhere is roughly equal, the surface temperature distribution of the welding area is uniform. The defective welding area, due to the large resistance of the defect, so that the loss of this area increases, the temperature increases. Through the infrared imaging equipment, can be a good infrared image, so as to accurately determine the hot spots, and then determine the existence of welding defects.

In the detection of welding defects are usually used AC heating, the benefits of this can be changed by changing the frequency of the power to control the penetration depth of the current. Low-frequency current penetration deeper, the discovery of internal defects beneficial; high-frequency current skin effect is strong, the surface temperature characteristics are more obvious.

\section{Machine vision technology and nondestructive testing}

\subsection{Overview of machine vision technology}

Machine vision is to use the machine instead of the human eye to do the measurement and judgment, it is in the semiconductor production, automobile manufacturing, pharmaceutical packaging and other industrial production process has been widely used. In the machine vision system, the machine vision product will be converted into the image signal into the image signal, transmitted to the image processing system, according to the pixel distribution and brightness, color and other information into digital signals, the image system for these signals to extract a variety of operations target characteristics, so as to get the target information of interest.

The composition of the human visual system is shown in Figure 5.1. The machine vision system is similar to the principle of the human eye vision system, and is also composed of signal acquisition, signal transmission, signal processing and other parts. Since the machine vision system is usually applied in industry, the industrial machine vision system usually contains a actuator.

The most important part of the machine vision system is the image processing and decision module, which can be logically divided into three stages: image preprocessing, feature extraction, pattern recognition and understanding. The preprocessing of an image is a process in which a low-quality digital image (a small contrast, a blurring and a deformation) obtained by an image forming apparatus is subjected to noise filtering, smoothing processing, image enhancement, and the like, which is easy to perform subsequent processing such as feature extraction. Image feature 
extraction is to extract the main feature quantity which is beneficial to image recognition and understanding from the bottom processed image. The characteristics of the original image are described by finite features. The features of the image include shape features, texture features, structural features, color features and fractal features. The main methods of feature extraction are regional segmentation, edge detection and texture analysis.

\subsection{Application of machine vision technology in steel plate defect monitoring}

Machine vision technology on-line nondestructive testing of steel plate surface defects originated in the early 1980 s. After entering the 1990s, machine vision technology based on linear array CCD device has undoubtedly become the mainstream technology of on-line detection of steel plate surface defects, and its application research work is in the ascendant.

Figure 5.3 is an actual steel plate surface machine vision detection system block diagram, is currently widely used steel plate defect detection system, including light source and image sensor subsystem, digital signal preprocessing subsystem, defect automatic classification subsystem, graphics display subsystem, quality analysis data statistics and database management subsystem, man-machine interface management subsystem. The specific implementation process has the following characteristics:

1. The use of standard digital CCD camera and lighting components, and the choice of specific technical indicators on the margin. At present, the universal use of pixels for the $2048 \sim 4096$ linear array CCD sensor unit, the illumination light source according to the specific surface of the steel plate to be tested, can be used high-intensity fluorescent lamp, array tungsten lamp or fiber optic light source.

2. Through the use of standardized bus structure and modular design technology, the system hardware structure common, flexible, stable and reliable operation, improvement and upgrading is very convenient.

3. Low-level visual computing tasks such as smoothing, enhancement, segmentation and description are done by dedicated processing components. The current research trend is to use high-speed DSP devices as processing components to complete the simple processing of images.

4. The traditional artificial intelligence methods (such as machine learning) based on symbolic system imitation of human intelligence and the method of simulating intelligence from the bottom of the biological system have been tried to solve the problem of automatic classification and identification of complex steel plate surface defects. But the existing defect automatic classifier only for some specific application background and specific types of defects show its effectiveness.

1. Image sensor 2, operator monitor 3, digital signal preprocessing 4, defect automatic classifier 5, graphic display subsystem 6 , system master module 7 , data analysis module 8 , image management subsystem 9 , man-machine interface A component 10, a network interface unit 11, a storage database system 12, and a quality control computer.

In general, the surface defect of steel plate machine vision detection system is a typical machine vision system, which collects the image information on the surface of the steel plate, through the processing of these image information, extract the specific information contained in these images, through these specific information to determine the surface of the steel plate defects, which is optoelectronic detection technology in the nondestructive testing of another application.

$\mathrm{X}$-ray is a kind of electromagnetic radiation similar to light, heat and radio waves, which is characterized by short wavelength (industrial X-ray detection commonly used in the wavelength range of about 0.1-0.001 nm). As the wavelength of the radiation material is shorter, the greater the ability to penetrate the material, so X-ray has a great ability to penetrate the material, it is the use of this feature for X-ray detection.

Figure 6.1 is a system diagram of a common X-ray inspection system in which X-rays are irradiated onto an object, and because it has a strong penetrating power, the lower detector can detect X-rays passing through the object, usually Will form an image, the image gray value and material, thickness and internal structure are closely related. It is precisely because of the image analysis can be obtained in the form of defects, so X-ray in the price of the internal testing has been widely used.

\subsection{Application of $\mathbf{X}$ - ray Inspection in Casting Defect Detection}

As the casting method has the advantages of low cost, one forming and can make complex structure large parts, it is widely used in many fields of industrial production, especially automobile manufacturing industry. In the aerospace industry, many parts are also castings. In order to ensure product quality and cost savings, it is necessary to detect defects in the early stages of the production process. Non-destructive testing is the preferred method of casting defect detection because it avoids material waste and increases production efficiency. So far, the most studied and effective methods include ultrasonic testing, X-ray radiography and ray tomography. 
With the expansion of production scale and quality testing standards, the radiation testing equipment and related technology requirements are getting higher and higher. For X-ray inspection, the new research focuses on increasing the acquisition of X-ray images, as well as the development of modern computer hardware and software technology to enable the detection process in high-speed and truly fully automated mode.

A commonly used X-ray inspection technology in the casting defect detection application is X-ray image automatic defect detection system. The system consists of four parts: a specimen support system; an X-ray generator; a sensor and an image processor that converts the X-ray energy into an electrical signal. Due to the particularity of the X-ray image, the corresponding sensor for obtaining the image is also different, there are two kinds: one is based on non-crystalline silicon or selenium sensor array flat grid; the other is based on linear diodes of the array.

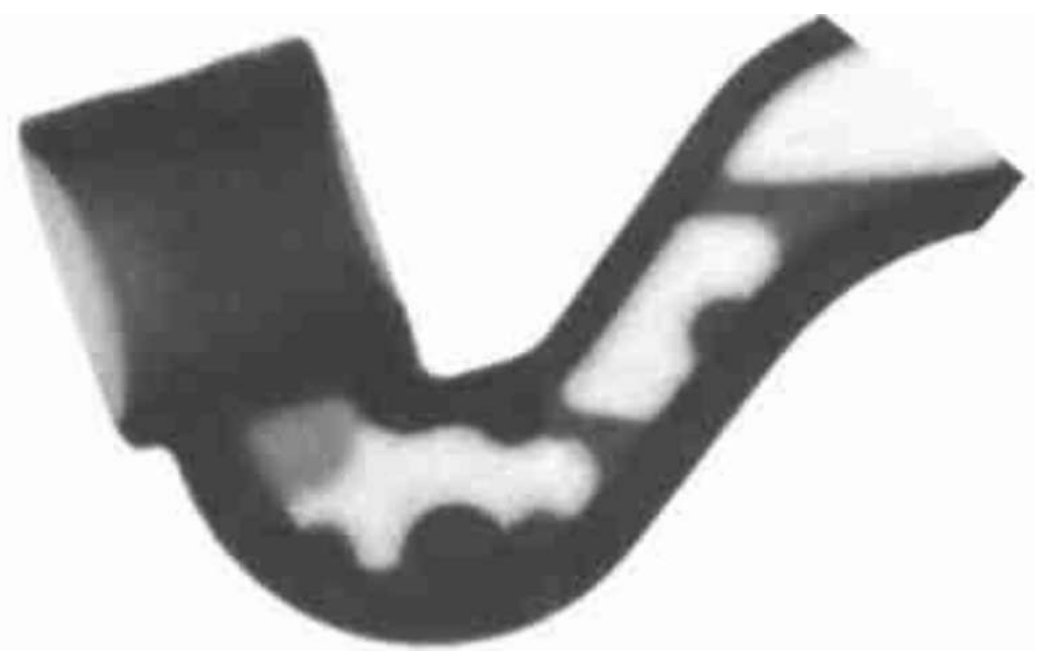

Figure 1. Picture of the castings obtained by X-ray inspection

Through the picture in Figure 6.2, after the follow-up image processing, you can get the type of defects in the casting, location and size.

$\mathrm{X}$-ray detection system is also a machine vision system, but the imaging method has changed, no longer through simple optical imaging to obtain defective images, but choose a new imaging method, so that some hidden inside the object defects exposed.

\section{Laser ultrasonic testing}

The laser can achieve non-contact high-sensitivity measurement, but cannot pass through the interior of the nontransparent material, and the ultrasonic detection method can achieve the internal quality of the test, so the laser excitation of ultrasonic waves through the test specimen, laser technology to receive this ultrasonic signal, the two together, the development of a new detection method - laser ultrasonic detection method to solve the conventional ultrasonic detection difficult to solve the problem.

Compared with the conventional ultrasonic detection method, laser ultrasonic technology has the following advantages: laser ultrasound does not require coupling agent, to avoid the coupling agent on the measurement range and accuracy of the impact; laser ultrasound can achieve long-distance operation can be used for high temperature environment and corrosive, radioactive and other harsh conditions, and can achieve rapid scanning, the rapid production of the scene of the work-piece on-line detection; laser ultrasound blind area is less than 100 microns, can be used to measure thin parts. The frequency of laser ultrasound is wider than that of conventional transducers and has the ability to measure tiny crack. Laser ultrasound can be used for complex and restricted geometrical shapes, such as small diameter pipes and so on. The spatial resolution is high. Which facilitates the precise positioning and dimensional measurement of defects and can be used as a sound source in theoretical research.

\subsection{Principles of laser ultrasonic testing}

Laser ultrasound is the use of high-energy laser pulse and the material surface of the instantaneous thermal effect, in the solid surface of the thermal characteristics of the area, and then use this small thermal layer in the material to the surrounding thermal expansion and diffusion of thermal stress, which through this thermal stress ultrasonic. Laser effect on the material to produce two thermal characteristics of the area: burning area, thermal bomb area. 


\section{Burning area}

As shown in Figure 7.1, under high energy, the temperature of the object rises beyond its evaporation temperature, and the atom exits the surface of the object at high speed, producing a momentum, which is called the thermal attack.

\section{Thermal bomb area}

As shown in Figure 7.2, when the energy of the laser is insufficient to form a corrosion phenomenon on the surface, a thermal characteristic region is generated on the solid surface, resulting in a stress wave, i.e., an ultrasonic wave, inside the object, and the heat absorbed by the surface does not exceed the melting temperature, the source of production is a brief expansion process, with the expansion of the pressure wave is mostly below the elastic range, this model is called the thermal effect.

Generally referred to as laser ultrasound refers to the thermal bomb area, because the excitation for the pulsed laser and the object surface is not required between the mechanical connection and contact, therefore, this method has a good engineering application potential and prospects. This method can fully realize non-contact ultrasonic testing when the optical method is used to receive the ultrasonic waves generated by the laser beam in the material to be tested.

\subsection{Laser Ultrasonic Testing Technology}

\section{Excitation laser}

Laser ultrasonic system is a set of light, machine, electricity, calculate the complex detection system, mainly composed of two parts, namely, the generation and reception of ultrasound. The laser currently produces ultrasonic waves are: a) Nd: YAG laser, which produces a laser wavelength of $1064 \mathrm{~nm}$, a laser energy of $300 \mathrm{~mJ}$, a laser spot diameter of $6.5 \mathrm{~mm}$, and a pulse width of $5 \mathrm{~ns}$; B) CO2 laser, the laser pulse duration of $70 \mathrm{~ns}$, according to the manufacturing needs, $\mathrm{CO} 2$ laser beam shape is rectangular, spot diameter of about $5 \mathrm{~mm}$; c) XeCL (308nm) laser, excitation pulse width is $40 \mathrm{~ns}$, XeCL laser and CO2 laser has a similar beam shape, spot diameter is about $3 \mathrm{~mm}$. In the detection of carbon fiber resin matrix composites, the composite materials have different absorption characteristics and ablation thresholds for these wavelengths of lasers. Nd: YAG lasers are more common.

\section{Optical acceptance technology}

(EMAT), capacitive transducer (MSAT) and air transducers, but the application of these non-contact optical detection methods in addition to their own limitations (Such as EMAT requirements for the test sample for the conductor; ESAT sample surface polishing and air transducers narrow bandwidth), the common feature is that although the non-contact, but the distance cannot be far away (a few millimeters to several Ten millimeters), the detection sensitivity decreases as the distance between the sample and the transducer increases. To achieve long-range non-contact detection can only use optical detection technology. Non-interfering blade technology requires that the sample surface is very smooth and difficult to use for rough surfaces. So in order to achieve long-range detection, commonly used optical interference technology, commonly used in the following two ways to achieve the interference of ultrasonic reception:

(1) Optical heterodyne technology

The heterodyne method is usually a Michelson interferometer, the surface of which is a mirror, and Figure 7.3 is a schematic of the heterodyne interferometer.

The response of the optical heterodyne method to the frequency is affected by the cutoff frequency of the detector, so the ultrasonic vibration greater than the cutoff frequency of the detector should be compensated by an electromechanical feedback loop to achieve this compensation effect. The heterodyne interferometry is sensitive to the displacement of the surface, but it cannot eliminate the interference of the various oscillations in the industrial field. In addition, this method has very harsh limits on the size of the spot, and only the spot size reaches a speckle size time to achieve the best detection results, so the optical heterodyne method can only be used for laboratory conditions under the smooth surface of the ultrasonic vibration detection, in the field detection is commonly used in the speed interferometer.

(2) Speed interferometer

The speed interferometer is also called a time delay interferometer, which is an optical detection method based on the Doppler shift principle.

When the laser is irradiated on the surface of the vibrating object, the frequency of the emitted or scattered light changes according to the principle of Doppler shift. The frequency of the ultrasonic wave is loaded in the frequency of change. The interferometer changes the frequency change into light intensity of the changes, through 
a series of processing means, converted into electrical signals displayed on the oscilloscope, the characteristics of the signal can be analyzed on the inside of the test specimen can be.

The speed interferometer is particularly sensitive to the speed of the surface of the detection object, and has good detection ability for micro vibration. This interferometer has dual-beam interference and multi-beam interference, with more of a confocal multi-beam interferometer, that is, FP interferometer, which has outstanding features better than other interferometers, Figure 7.5 shows the relationship between the light intensity of the light in the interferometer and the intensity of the output signal.

Using a working system shown in Figure 7.6, a Q-switched Nd: YAG laser was used to hit the test specimen. A He-Ne laser was used to receive the generated ultrasonic waves on the other side of the sample. The signal was detected by the carrier ultrasonic frequency of the laser beam frequency shift, the use of multi-beam interference characteristics, the frequency changes into the output light intensity changes, in order to achieve the optical method of ultrasonic testing.

\section{Conclusion}

In this paper, the application of photoelectric detection technology in non-destructive testing of mechanical components is introduced, including infrared imaging technology, machine vision technology, X-ray nondestructive testing technology and laser ultrasonic detection technology. There are many similarities between the four, such as the four methods are to obtain the sample of the 'image', through the analysis of the image analysis, access to information contained in the information, and this information is the sample defect information, there are many similarities between the four image processing techniques used.

$\mathrm{X}$-ray detection technology is mainly to obtain parts of the internal defects, machine vision technology is more access to the surface of the defective items, and infrared imaging technology can not only get internal defects, but also access to surface defects. There are some differences between the four ways to obtain images, X-ray is through the ability of the penetration of the ray to obtain the image, the machine vision technology through the optical sensor to obtain the optical characteristics of objects, infrared imaging technology through the infrared radiation characteristics of objects to get the image.

It is because of the photoelectric detection technology has non-contact, high precision, high speed and so on, so in modern non-destructive testing technology has been widely used, and the scope of application is gradually expanding.

\section{$\underline{\text { References }}$}

1. Guo Jiayuan, et al. Photoelectric detection technology and application [M]. Beijing University of Aeronautics and Astronautics, 2006.

2. Zhong Liyun. Development and application of photoelectric detection technology [J]. Laser Magazine, 2000.

3. Zhou Jinlong. New fiber grating technology and its application in optical communication and optical fiber sensing [D]. Xiamen University, 200.

4. Wang Yi, et al. Research and design of a new type of photoelectric detection circuit [J]. Optoelectronic Technology, 2012,32 (2): 131-136.

5. Zhu RenSheng, Qu Bo. Photoelectric sensor and its application [J]. Electric Age, 2010 (4): 111-113.

6. Li Zhiquan et al. Research on signal demodulation technology of fiber grating sensing system [J]. Journal of Applied Optics, 2005,26 (4): 36-41.

7. Feng Mengyun, Huang Xiaqing. Based on intelligent photoelectric detection circuit design [J]. Association for Science and Technology, 2012, (7)

8. Li Fei, Xu Jin. A new DNA self-assembly magnetic bead photoelectric detection system and its application in the development of DNA computer [J] .Journal of Computer Science, 2013,36 (9)

9. Du Liting, Liu Songping. Laser ultrasonic detection technology [J]. Non-destructive testing .2011.35 (5): 1-4.

10. YANG Li-feng, WANG Ya-fei.Application of Laser in Ultrasonic Testing [J] .Progress in Laser and Optoelectronics .2006.43 (2): 29-32.

11. Sun Lina, original Peixin. X-ray security equipment in the detection technology research [J]. China Testing Technology, 2006,32 (3): 20-23. 